\title{
Red Organic Light-emitting Diodes utilizing Energy Transfer and Charge Trapping
}

\author{
Ju-Seung Kim ${ }^{\mathrm{a}}$ \\ Center for Advanced Science and Innovation, Osaka University, \\ Yamadaoka, Suita Osaka, 565-0871, Japan \\ Hal-Bon $\mathrm{Gu}$ \\ Department of Electrical Engineering, Chonnam National University, \\ Yongbong-dong, Buk-gu, Gwangju 500-757, Korea \\ ${ }^{a}$ E-mail : jskim@crcast.osaka-u.ac.jp
}

(Received March 15 2005, Accepted June 7 2005)

\begin{abstract}
We report the efficient red light-emitting diodes based on the fluorescent dye 4 (dicyanomethylene)-2-i-propyl-6-(1,1,7,7-tetramethyljulolidyl-9-enyl)-4H-pyran (DCJTI) and 5,6,11,12-tetraphenyl naphthacene (rubrene) codoped in the tris(8-hydroxyquinoline)aluminum $\left(\mathrm{Alq}_{3}\right)$. Luminance efficiency of $2.2 \mathrm{~cd} / \mathrm{A}$ with a Commission International De L'Eclairage (CIE) chromaticity coordinate of $\mathrm{x}, \mathrm{y}=(0.640,0.350)$ are achieved at the driving current density of 20 $\mathrm{mA} / \mathrm{cm}^{2}$. Adding the rubrene to the DCJTI in tris $\left(8\right.$-hydroxyquinoline)aluminum $\left(\mathrm{Alq}_{3}\right)$, the red color purity and luminance efficiency improved comparing to the DCJTI only doped devices because the rubrene molecules assist the polarization effect of DCJTI by molecular interaction and enhance the energy transfer from $\mathrm{Alq}_{3}$ to DCJTI.
\end{abstract}

Keywords: OLED, Carrier trapping, Energy transfer, Emitting assist dopant

\section{INTRODUCTION}

During the past several years, organic light-emitting diodes (OLEDs) utilizing low-molecular dyes and polymer materials have been widely studied because of their possible application to the full-color display panel. Realizing an OLED for practical use, however, have some obstacles to overcome containing the limits of luminous efficiency especially in red color[1,2]. It is difficult to achieve the high luminance with pure red emission in typical red OLEDs because of low fluorescent efficiency and concentration quenching in the emitting layer. In order to prevent the concentration quenching and improve the luminous efficiency, doping system is mainly utilized to obtain the red emission from the emitting layer, which consist with a host material and red guest fluorescent dyes. Tris(8-hydroxyquinoline) aluminum $\left(\mathrm{Alq}_{3}\right)$ is widely used as the host material because the emission from $\mathrm{Alq}_{3}$ sufficiently overlaps with the absorption of red dyes as well as its charge transport ability[3]. However, it is still remaining the difficulty to achieve the high luminous efficiency with pure red emission because the energy transfer is not complete between the host and guest materials resulting the emission color change to orange region and showing the concentration quenching at high doping concentration. Recently, two-step energy transfer using the emission assist dopant is demonstrated to improve the emission properties of red OLEDs[4,5].

Although a number of new prospective red fluorescent dyes have been synthesized, 4-(dicyanomethylene)-2-tbutyl-6-(1,1,7,7-tetramethyljulolidyl-9-enyl)-4H-pyran (DCJTB) is still the most efficient material showing an excellent charge transport and high emitting efficiency [6]. Moreover, DCJTB is considered as a best red guest material in doped $\mathrm{Alq}_{3}$. In terms of practical use, the red color emission recommended to reach to the Commission International De L'Eclairage (CIE) chromaticity coordinate of $x, y=(0.640,0.350)$. Feng Li et al[7] reported that CIE coordinate $x, y=(0.64,0.35)$ is can be achieved by adding $5 \%$ of rubrene as an emitting assist dopant to the $3 \%$ DCJTB in doped $\mathrm{Alq}_{3}$ at $18 \mathrm{~V}$ and T. H. Liu et al[6] showed the luminance efficiency of $3.24 \mathrm{~cd} / \mathrm{A}$ with CIE coordinate $\mathrm{x}, \mathrm{y}=(0.643,0.354)$ at current density of $20 \mathrm{~mA} / \mathrm{cm}^{2}$ accomplished by increasing the thickness of hole transport layer and replacing copper phthalocyanine $(\mathrm{CuPc})$ with $\mathrm{CHF}_{3}$ plasma polymerized hole injection layer. 
In spite of these progressive results, utilizing the DCJTB as a red emitting material is one of the factor to prohibit the realization of OLEDs in practical use because the cost of manufacturing DCJTB is high owing to the synthetic complication. There are some efforts to simplify the synthetic process of a key intermediate, 1,1,7,7-tetramethyljulolidine (TMJ), involved in the synthesis of DCJTB [8]. C. H. Chen et al[9] reported that the isopropyl substituted red fluorescent dye, 4(dicyanomethylene)-2-i-propyl-6-(1,1,7,7-tetramethyljulolidyl-9-enyl)-4H-pyran (DCJTI), can be replace the DCJTB because DCJTI may prove to be cheaper than DCJTB through changing synthesis of the key intermediate.

In this study, we fabricate the red OLEDs varying with the doping concentration of DCJTI and rubrene doped in $\mathrm{Alq}_{3}$ for achieving the high efficiency with saturated red emission having CIE coordinate $\mathrm{x}, \mathrm{y}=(0.64$, $0.35)$.

\section{EXPERIMENTAL}

For the optical absorption and photoluminescence (PL) measurement, $\mathrm{Alq}_{3}$, rubrene, and DCJTI contained chloroform solutions were prepared. Absorption and PL spectra were measured with UV-VIS-NIR scanning spectro-photometer (Shimadzu, UV-3100FS) and spectroflurometer(Jasco, FP-6500), respectively.

The device structure which consisted with ITO/ $\alpha-$ $\mathrm{NPD}(40 \mathrm{~nm}) / \mathrm{Alq}_{3}$ :rubrene:DCJTI $(30 \mathrm{~nm}) / \mathrm{BCP}(10 \mathrm{~nm}) /$ $\mathrm{Alq}_{3}(20 \mathrm{~nm}) / \mathrm{LiF}(0.5 \mathrm{~nm}) / \mathrm{Al}(50 \mathrm{~nm})$ and the molecular structures of materials are shown in Fig. 1. In this device, $\alpha$-NPD is used as the hole transport layer, $\mathrm{Al}_{3}$ doped with rubrene, and DCJTI as the emissive layer, in addition, BCP layer introduced as an exciton and hole blocking layer. Before the evaporation of organic materials, the indium tin oxide (ITO) coated glass substrate was thoroughly cleaned by sonication with acetone, ethanol, and chloroform and treated by $\mathrm{UV}-\mathrm{O}_{3}$ cleaner. All organic layers were prepared by the organic molecular beam deposition (OMBD) method at a vacuum level of $2 \times 10^{-7}$ Torr and the deposition rate of the organic films maintained at $0.5-1 \AA / \mathrm{s}$. The doping concentration of DCJTI controlled from 0.3 to $5 \mathrm{v} / \mathrm{v} \%$ changing with various ratio, and that of rubrene relative to the $\mathrm{Alq}_{3}$. The doping concentration rates of DCJTI and rubrene in $\mathrm{Alq}_{3}$ were controlled by monitoring evaporation rates of each material. After evaporating of organic layer, $0.5 \mathrm{~nm}$ - thick lithium fluoride $(\mathrm{LiF})$ and $50 \mathrm{~nm}$ - thick Al electrodes were sequentially deposited at $2 \times 10^{-4} \mathrm{~Pa}$ on the top of organic layer. The deposition rates of $\mathrm{LiF}$ and $\mathrm{Al}$ were $1 \AA / \mathrm{s}$, respectively. For protecting the metal electrode from oxidation during the device evaluation, $100 \mathrm{~nm}$-thick $\mathrm{Ag}$ also evaporated on the top of the $\mathrm{Al}$ electrode. The active emitting area is 4 $\mathrm{mm}^{2}$. Finally, the device was covered with a glass plate and sealed by epoxy resin in an Ar gas filled dry box.

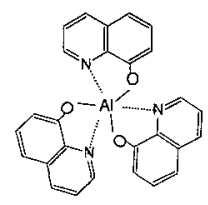

$\mathrm{Alq}_{3}$

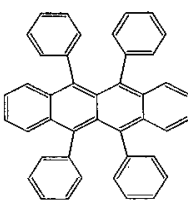

rubrene

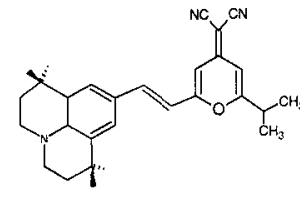

DCJTI

\begin{tabular}{|c|}
\hline $\mathrm{Al}$ \\
\hline $\mathrm{LiF}$ \\
\hline $\mathrm{Alq}_{3}$ \\
\hline $\mathrm{BCP}$ \\
\hline $\begin{array}{c}\mathrm{Alq}_{3}: \text { Rubrene } \\
: \mathrm{DCJTI}\end{array}$ \\
\hline$\alpha-$ NPD \\
\hline ITO \\
\hline Glass Substrate \\
\hline
\end{tabular}

Fig. 1. Molecular structures of organic materials and configuration of device used in this study.

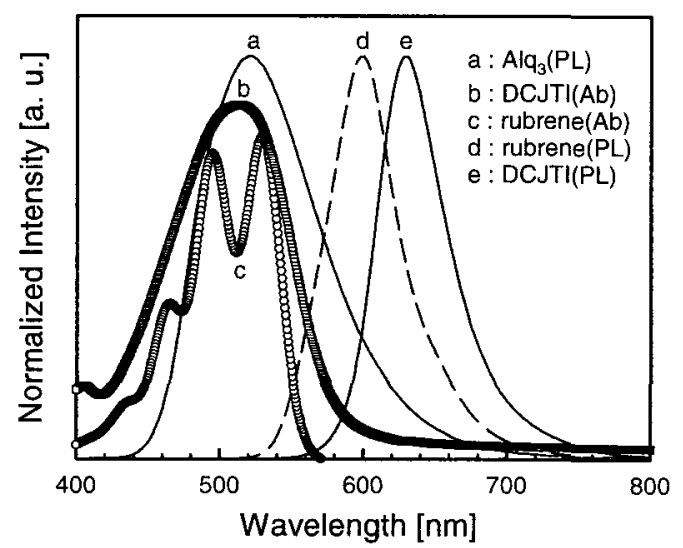

Fig. 2. Absorption and PL spectra of $\mathrm{Alq}_{3}$, rubrene, and DCJTI in chloroform solution.

The voltage-current-luminance characteristics were estimated by Labview controlled DC power supply (Kenwood PW36-1), multimeter (Keithley 2000), and luminance meter (Minolta LS-100). The CIE chromaticity coordination and electroluminescence (EL) spectra were measured simultaneously by photonic multi-channel analyzer (Hamamatsu PMA-11).

\section{RESULTS AND DISCUSSION}

Figure 2 shows the PL spectrum of $\mathrm{Alq}_{3}$, and optical absorption and PL spectra of rubrene and DCJTI, respectively. The solution of rubrene has the absorption maximum at the wavelength of $529 \mathrm{~nm}$ with a shoulder 
peak at $495 \mathrm{~nm}$ and DCJTI has the peak at the wavelength of $513 \mathrm{~nm}$. The PL spectra of $\mathrm{Alq}_{3}$, rubrene, and DCJTI show the wavelength at 520, 599, and 629 $\mathrm{nm}$, respectively. According to the förster energy transfer theory, we expect that the efficient energy transfer from $\mathrm{Alq}_{3}$ to rubrene and DCJTI occurs in the emissive layer because of high degree of overlap area in absorption of DCJTI and PL of $\mathrm{Alq}_{3}$ as well as the absorption of rubrene and PL spectrum of $\mathrm{Alq}_{3}$.

In the first place, we fabricated the DCJTI only doped in $\mathrm{Alq}_{3}$ device changing with the various concentration of DCJTI. Figure 3 shows the EL spectra as a function of the doping concentration of DCJTI from 0.3 to $5 \%$ at a current density of $20 \mathrm{~mA} / \mathrm{cm}^{2}$. With increasing doping ratio of the DCJTI, we observed that the main peak of the EL spectra is moved to longer wavelength. This spectral shift is attributed to solid-state solvation effect of the DCJTI molecule when it doped in $\mathrm{Alq}_{3}$ which is strong polar host. V. Bulovic et al[10] reported the same phenomena on the 4-(dicyanomethylene)-2-methyl-6-p(dimethylamino)styryl-4H-pyran (DCM) doped in $\mathrm{Alq}_{3}$. In EL spectrum of the DCJTI doped in $\mathrm{Alq}_{3}$ at concentration of $0.3 \%$, the side peak of $520 \mathrm{~nm}$ is clearly appeared. This side peak is originated from $\mathrm{Alq}_{3}$ and represent the energy transfer from $\mathrm{Alq}_{3}$ to DCJTI is not complete. With increasing the concentration of DCJTI, the side peak is gradually decreased and it seems to disappear at the higher concentration. Even the concentration of 2 and $3 \%$, however, the side peak is still remained at the wavelength of $520 \mathrm{~nm}$ (shown in the inset of Fig. 3). We find that the concentration of DCJTI increases to $5 \%$, the emission peak of $\mathrm{Alq}_{3}$ disappeared. The luminous efficiency of the devices is decreased with increasing the doping concentration of DCJTI. This concentration dependent EL spectra and efficiency understood by the energy transfer from the $\mathrm{Alq}_{3}$ to the DCJTI molecules and concentration quenching of DCJTI due to the intermolecular interaction[11]. When the $5 \%$ DCJTI doped in $\mathrm{Alq}_{3}$, we obtained the CIE coordinate $\mathrm{x}$, $y=(0.647,0.347)$ at a current density of $20 \mathrm{~mA} / \mathrm{cm}^{2}$ while the luminous efficiency is $0.5 \mathrm{~cd} / \mathrm{A}$ at a same current density. Although the chromaticity coordinates is sufficiently satisfy the initial goal of this work, the luminous efficiency is showed significantly lower value compared to the previous reports[6,7].

To achieve the high luminous efficiency with pure red emission, rubrene, which called emitting assist dopant is codoped to the emitting layer. As shown in the Fig. 3, the energy transfer from $\mathrm{Alq}_{3}$ to DCJTI was not completed in 2 and $3 \%$ doping concentration of DCJTI. Therefore, we observed the doping effect of rubrene on the device used the 2 and $3 \%$ doping concentration of DCJTI.

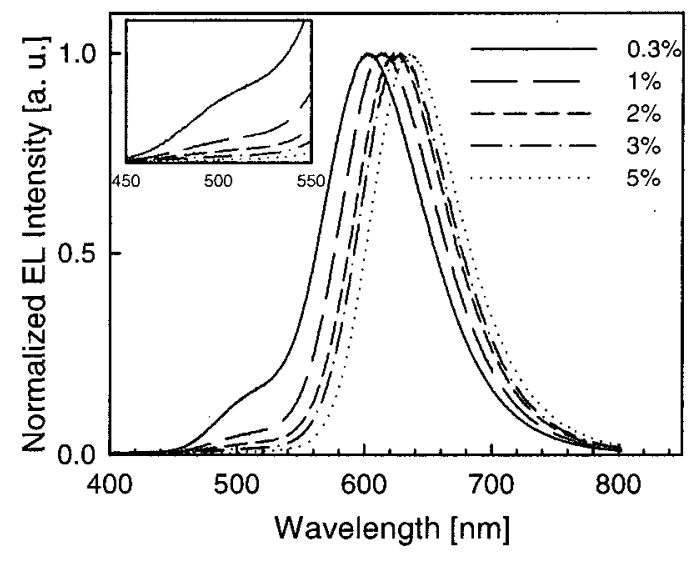

Fig. 3. Normalized EL spectra as a function of the doping concentration of DCJTI at a current density of 20 $\mathrm{mA} / \mathrm{cm}^{2}$. The inset indicated the part of spectra to identify the emission from $\mathrm{Alq}_{3}$.

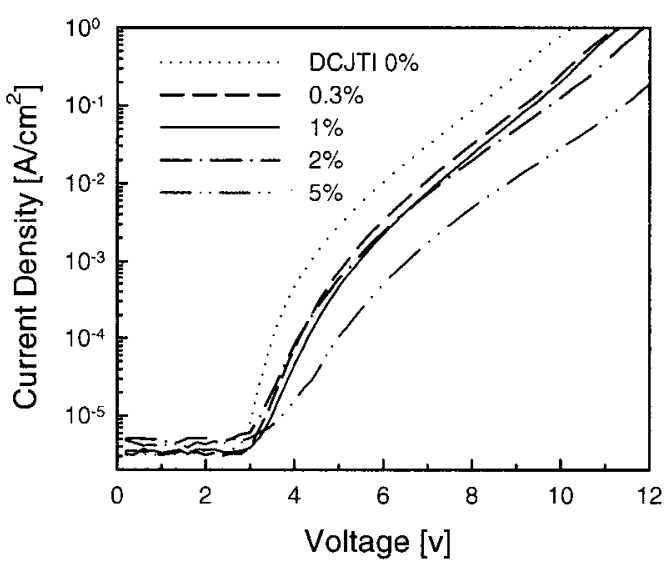

(a)

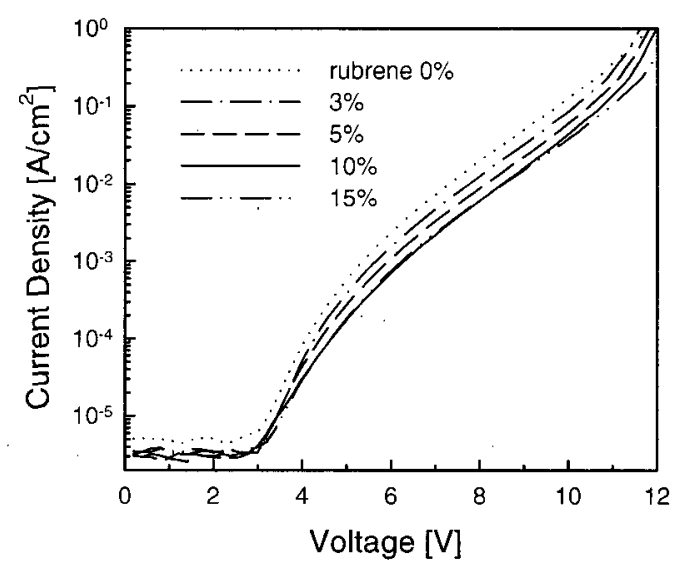

(b)

Fig. 4. Voltage-current (V-I) characteristics of (a) DCJTI only doped in $\mathrm{Alq}_{3}$, and $\operatorname{DCJTI}(2 \%)$ and rubrene codoped OLEDs. 


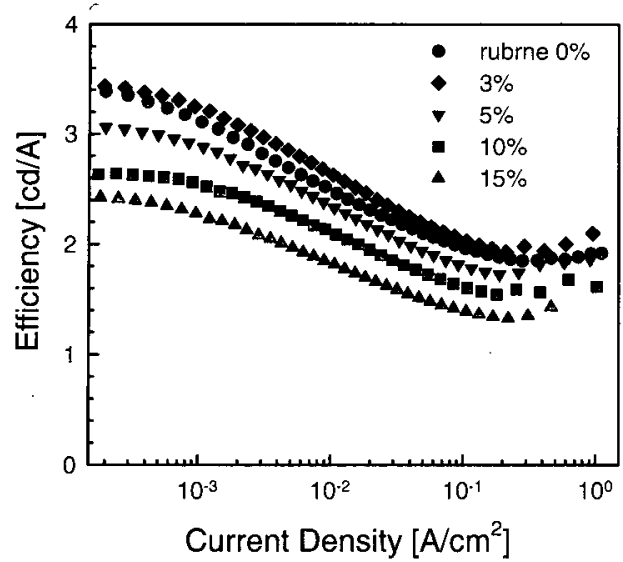

Fig. 5. Current-luminous efficiency as a function of doping concentration of rubrene codoped with DCJTI $2 \%$ in $\mathrm{Alq}_{3}$.

To understand the doping influence of the rubrene to the DCJTI doped in $\mathrm{Alq}_{3}$ emitting layer with regard to the carrier transport and the emitting processes, we investigated the voltage-current density (V-I) characteristics of ITO/ $\alpha-\mathrm{NPD} / \mathrm{Alq}_{3}: \mathrm{DCJTI}:$ rubrene/BCP/Alq $3 /$ $\mathrm{LiF} / \mathrm{Al}$ devices with the different rubrene concent-ration. Figure 4 shows the V-I characteristics of ITO/ $\alpha$ $\mathrm{NPD} / \mathrm{Alq}_{3}: \mathrm{DCJTI} / \mathrm{BCP} / \mathrm{Alq}_{3} / \mathrm{LiF} / \mathrm{Al}$ devices with changing the DCJTI concentrations and ITO/ $\alpha-\mathrm{NPD} / \mathrm{Alq}_{3}: \mathrm{DCJTI}$ : rubrne/BCP/Alq ${ }_{3} / \mathrm{LiF} / \mathrm{Al}$ devices at various rubrene concentrations on $2 \%$ DCJTI. In the Fig. 4(a), the current density is significantly decreased with increasing the DCJTI doping concentration in $\mathrm{Alq}_{3}$ when the forward bias applied. The electron mobility in $\mathrm{Alq}_{3}$ and BCP layer were determined $4.7 \times 10^{-6}$ and $1.1 \times 10^{-3}$ $\mathrm{cm}^{2} / \mathrm{Vs}$ at electric field of $1 \times 10^{6} \mathrm{~V} / \mathrm{cm}$, respectively and the hole mobility in $\alpha$-NPD is in the range between $10^{-4}$ and $10^{-3} \mathrm{~cm}^{2} / \mathrm{Vs}$ at electric filed of $10^{5} \mathrm{~V} / \mathrm{cm}[12,13]$.

Considering the carrier mobility in organic layer of our devices, the current flow can be limited by the electron mobility in the $\mathrm{Alq}_{3}$ and doped $\mathrm{Alq}_{3}$ layer. Therefore, the

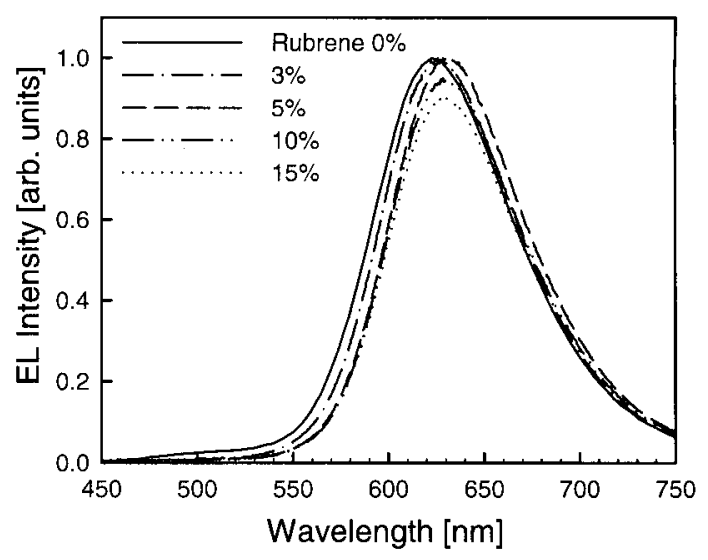

Fig. 6. EL spectra of red OLEDs as a function of the rubrene doping concentration (DCJTI $2 \%$ ).

strong DCJTI concentration dependence of the V-I characteristics indicate that the DCJTI molecules doped in $\mathrm{Alq}_{3}$ play the role of charge carrier trapping sites in the process of EL. In the Fig. 4(b), the current is slightly decreased with increasing the rubrene doping concentration to the DCJTI doped in $\mathrm{Alq}_{3}$ layer. V-I characteristics according to the rubrene doping concentration is less dependent comparing with DCJTI only doped devices. These results indicate that the doped rubrene act as shallow carrier trapping site as well as the emitting assistant material for energy transfer.

Figure 5 shows the current-luminous efficiency as a function of doping concentration of rubrene codoped to the $2 \%$ DCJTI in $\mathrm{Alq}_{3}$. As increasing current density, the device efficiency drops gradually due to the exciton quenching. The small increasing at high current density is attributed to the introducing of BCP layer. When the $3 \%$ rubrene doped to the emitting layer, the luminous efficiency slightly increased because of energy transfer and carrier trapping effects of rubrene molecules. With the increasing the doping concentration of rubrene, however, the luminous efficiency is gradually decreased

Table 1. EL performance of the DCJTI and rubrene codoped red OLEDs in various doping concentration at applied current density of $20 \mathrm{~mA} / \mathrm{cm}^{2}$.

\begin{tabular}{|c|c|c|c|c|c|}
\hline \multicolumn{2}{|c|}{$\begin{array}{c}\text { Doping Concentration } \\
(\mathrm{v} / \mathrm{v} \%)\end{array}$} & \multirow{2}{*}{$\begin{array}{l}\text { Turn on } \\
\text { voltage } \\
\text { (V) }\end{array}$} & \multirow{2}{*}{$\begin{array}{l}\text { Luminance } \\
\left(\mathrm{cd} / \mathrm{m}^{2}\right)\end{array}$} & \multirow{2}{*}{$\begin{array}{l}\text { Efficiency } \\
(\mathrm{cd} / \mathrm{A})\end{array}$} & \multirow{2}{*}{$\begin{array}{c}\mathrm{CIE} \\
(\mathrm{x}, \mathrm{y})\end{array}$} \\
\hline DCJTI & rubrene & & & & \\
\hline \multirow[t]{3}{*}{2} & 0 & 8 & 460 & 2.3 & $0.617,0.374$ \\
\hline & 3. & 8.5 & 490 & 2.4 & $0.628,0.366$ \\
\hline & 5 & 8.9 & 435 & 2.2 & $0.640,0.355$ \\
\hline \multirow[t]{3}{*}{3} & 0 & 8.5 & 251 & 1.3 & $0.630,0.363$ \\
\hline & 3 & 9.3 & 340 & 1.7 & $0.646,0.349$ \\
\hline & 5 & 10.6 & 304 & 1.6 & $0.645,0.351$ \\
\hline
\end{tabular}


due to the concentration quenching. The red emission purity of the device with $5 \%$ rubrene is better than that of the device with $3 \%$ rubrene.

Figure 6 shows the EL spectra with different dopping concentration of rubrene codoped to the $2 \%$ DCJTI in $\mathrm{Alq}_{3}$. We found that the maximum emission peak shifted from 624 to $632 \mathrm{~nm}$ with increasing the rubrene concentration of 0 to $5 \%$ and then slightly decreased to $628 \mathrm{~nm}$ during the rubrene concentration increase to $15 \%$. Moreover, CIE coordinates $\mathrm{x}$ and $\mathrm{y}$ values also gradually changed from $\mathrm{x}, \mathrm{y}=(0.617,0.374)$ to $(0.640$, $0.355)$ and moved to $(0.637,0.359)$ at the same concentration of rubrene. These results can be explained by molecular polarization effect, in addition to the energy transfer.between the rubrene molecules and the DCJTI molecules. The side peak from $\mathrm{Alq}_{3}$ at $520 \mathrm{~nm}$ is disappeared with adding the only $3 \%$ of rubrene and the peak wavelength at $600 \mathrm{~nm}$, which originated rubrene, was not observed. These results mean that the rubrene enhance the energy transfer rate from $\mathrm{Alq}_{3}$ to DCJTI and the energy transfer is not saturated even adding the rubrene concentration of $15 \%$.

The electrical and optical characteristics of red OLEDs with various. DCJTI and rubrene doping concentration in $\mathrm{Alq}_{3}$ at current density of $20 \mathrm{~mA} / \mathrm{cm}^{2}$ are shown in Table 1. It is can be seen that the operation voltage is slightly increased with increasing with doping concentration of DCJTI and rubrene. These results represent that the DCJTI and rubrene molecules act as trapping site when they codoped in $\mathrm{Alq}_{3}$. In terms of the red color purity as well as luminance efficiency, $2 \%$ DCJTI with $5 \%$ rubrene codoped in $\mathrm{Alq}_{3}$ device shows the optimal characteristics showing the luminance efficiency of $2.2 \mathrm{~cd} / \mathrm{A}$ and CIE coordinates $\mathrm{x}, \mathrm{y}=(0.640$, $0.355)$.

\section{CONCLUSION}

We have fabricated the DCJTI and rubrene codoped red OLEDs where a luminance efficiency of $2.2 \mathrm{~cd} / \mathrm{A}$ with CIE coordinates of $x, y=(0.640,0.355)$ at current density of $20 \mathrm{~mA} / \mathrm{cm}^{2}$ and driving voltage was achieved at $8.9 \mathrm{~V}$ with initial device luminance of $435 \mathrm{~cd} / \mathrm{m}^{2}$. Adding the rubrene molecule as an emitting assist dopant, the efficient red OLEDs were obtained with deeply saturated red emission color purity. Rubrene molecules, codoped with DCJTI in $\mathrm{Alq}_{3}$ host, supplied the additional carrier trapping site and enhanced the energy transfer from $\mathrm{Alq}_{3}$ host to DCJTI guest. We also confirm that the DCJTI is alternative of the prospective red emitting material to fabricate the full color OLEDs.

\section{ACKNOWLEDGMENTS}

This work was supported by the RRC-HECS, Chonnam National University.

\section{REFERENCES}

[1] J. Kido, H. Hayase, K. hongawa, K. Okuyama, K. Nagai, and K. Okuyama, "Bright red light-emitting organic electroluminescent devices having a europium complex as an emitter", Appl. Phys. Lett., Vol. 64, p. 815, 1994.

[2] R. A. Campos, I. P. Kovlev, Y. Guo, N. Wakili, and T. Skotheim, "Red electroluminescence from a thin organometallic layer of europium", J. Appl. Phys., Vol. 80, p. 7144, 1996.

[3] Z. Y. Xie, L. S. Hung, and S. T. Lee, "Highefficiency red electroluminescence from a narrow recombination zone confined by an organic double heterostructure", Appl. Phys. Lett., Vol. 79, p. 1048, 2001.

[4] Y. Hamada, H. Kanno, T. Tsujioka, H. Takahashi, and T. Usuki, "Red organic light-emitting diodes using an emitting assist dipant", Appl. Phys. Lett., Vol. 75, No. 12, p. 1682, 1999.

[5] J. Feng, F. Li, W. Gao, G. Cheng, W. Xie, and S. Liu "Improvement of efficiency and color purity utilizing two-step energy transfer for red organic light-emitting devices", Appl. Phys. Lett., Vol. 81, p 2935, 2002.

[6] T.-H. Liu, C.-Y. Iou, S.-W. Wen, and C. H. Chen, "4(Dicyanomethylene)-2-t-butyl-6-(1,1,7,7-tetramethyljulolidyl-9-enyl)-4H-pyran doped red emitters in organic light-emitting devices", Thin Solids Films, Vol. 441, p. 223, 2003.

[7] F. Li, J. Lin, J. Feng, G. Cheng, H. Liu, S. Liu, L. Zhang, X. Zhang, and S. T. Lee, "Electrical and optical characteristics of red organic light-emitting diodes doped with two guest dyes", Synth. Met., Vol. 139, p. 341, 2003.

[8] B. Balaganesan, S.-W. Wen, and C. H. Chen, "Synthetic study of tetramethyljulolidine - a key intermediate toward the synthesis of the red dopant DCJTB for OLED application", Tetrahedron Letters Vol. 44, p. 145, 2003.

[9] C. H. Chen, C. W. Tang, J. Shi, and K. P. Klubek, "Recent developments in the synthesis of red dopants for $\mathrm{Alq}_{3}$ hosted electroluminescence", Thin Solid films, Vol. 363, p. 327, 2000.

[10] S. V. Bulovic, R. Deshpande, M. E. Thompson, and S. R. Forrest, "Tunning the color emission of thin film molecular organic light emitting devices by the solid state solvation effect", Chem. Phys. Lett., Vol. 308, p. 317, 1999.

[11] R. N. Bera, Y. Sakakibara, M. Tokumoto, and K. Yase, "Time-redolved photoluminescence study on energy transfer from $\mathrm{Alq}_{3}$ (tris(8-hydroxyquinoline) aluminum) to red emissive tetraphenylchlorin", Jpn 
J. Appl. Phys., Vol. 42, No.12, p. 7379, 2003.

[12] T. Yasuda, Y. Yamaguchi, De-Chun Zou, and T. Tsutsui, "Carrier mobilities in organic electron transport materials determined from space charge limited current”, Jpn. J. Appl. Phys., Vol. 41, No. 9, p. 5626, 2002.

[13] S. Naka, H. Okada, H. Onnagawa, Y. Yamaguchi, and T. Tsutsui, "Carrier transport properties of organic materials for EL device operation", Synth. Met., Vol. 111-112, p. 331, 2000. 